\begin{tabular}{|c|l|}
\hline Title & Surface Fermi-level position and gap state distribution of InGaP surface grown by metalorganic vapor-phase epitaxy \\
\hline Author(s) & Hashizume, Tamotsu \\
\hline Citation & $\begin{array}{l}\text { A pplied Physics Letters, 81/13), 2382-2384 } \\
\text { https://doi.org/10.1063/1509119 }\end{array}$ \\
\hline Issue Date & 2002-09-23 \\
\hline Doc URL & http://hdl.handle.net/2115/5541 \\
\hline Rights & Copyright $\odot 2002$ A merican Institute of Physics \\
\hline Type & article \\
\hline File Information & APL81-13.pdf \\
\hline
\end{tabular}

Instructions for use 


\title{
Surface Fermi-level position and gap state distribution of InGaP surface grown by metalorganic vapor-phase epitaxy
}

\author{
Tamotsu Hashizume ${ }^{\text {a) }}$ \\ Research Center for Integrated Quantum Electronics (RCIQE), Hokkaido University, Sapporo 060-8628, \\ Japan
}

(Received 3 June 2002; accepted for publication 30 July 2002)

\begin{abstract}
Electronic properties of "free" $n$ - $\operatorname{In}_{0.49} \mathrm{Ga}_{0.51} \mathrm{P}$ surfaces grown by metalorganic vapor-phase epitaxy were directly characterized using the contactless capacitance-voltage technique. The HCl-treated surface showed a wide and continuous distribution of surface state density $\left(D_{\mathrm{ss}}\right)$ in energy with relatively low densities, leading to no pronounced Fermi-level pinning effect on the surface. The minimum $D_{\text {ss }}$ value was determined to be $8 \times 10^{11} \mathrm{~cm}^{-2} \mathrm{eV}^{-1}$. The surface Fermi-level position was found at $1.2 \mathrm{eV}$ above the valence band maximum, consistent with the $\mathrm{x}$-ray photoelectron spectroscopy results. (C) 2002 American Institute of Physics. [DOI: 10.1063/1.1509119]
\end{abstract}

InGaP/GaAs-based heterojunction bipolar transistors (HBTs) and pseudomorphic high-electron mobility transistors are establishing their status as key devices in a wide variety of microwave and optical communication systems at a rapid pace. One of the reasons for significant improvements of reliability in InGaP/GaAs-based devices may be related to better surface properties of InGaP than AlGaAs. In fact, the remarkable suppression of frequency dispersion of the drain current in power GaAs field-effect transistors (FETs $)^{1}$ and the reduction of surface recombination current in $\mathrm{InGaP} / \mathrm{GaAs} \mathrm{HBTs},{ }^{2}$ were achieved using a InGaP passivation layer. Furthermore, an InGaP passivation ledge process led to the long-term reliability in InGaP/GaAs HBTs., However, the mechanism of such passivation effects is not fully understood. In addition, a variety of surface atomic arrangements of InGaP due to the well-known sublattice ordering effects ${ }^{5-7}$ make the control of surfaces and heterointerfaces rather complicated and difficult. Nevertheless, experimental confirmation on the electronic properties of "free" InGaP surfaces, in particular, densities and distributions of surface states, has been largely lacking.

This letter presents the characterization of surface electronic properties of "free" InGaP surfaces using a contactless capacitance-voltage $(C-V)$ technique in ultrahigh vacuum (UHV) condition. In combination with $\mathrm{x}$-ray photoelectron spectroscopy (XPS) measurements, the surface Fermi-level position and the surface state density distribution were evaluated.

A sample used in the present study was a Si-doped $\mathrm{In}_{0.49} \mathrm{Ga}_{0.51} \mathrm{P}$ epitaxial layer grown on an $n^{+}$-GaAs $(001)$ substrate at $580{ }^{\circ} \mathrm{C}$ by metalorganic vapor-phase epitaxy (MOVPE) using trimethylindium, triethylgallium, and phosphine as precursers. The layer thickness of InGaP was approximately $0.6 \mu \mathrm{m}$. An electron concentration of the grown layer was determined to be $6 \times 10^{16} \mathrm{~cm}^{-3}$ by a Schottky $C-V$ method. All the sample surfaces were cleaned in organic solvents. Then, some samples were treated in $\mathrm{HCl}: \mathrm{H}_{2} \mathrm{O}=1: 2$ solution and in $\mathrm{HF}: \mathrm{C}_{2} \mathrm{H}_{5} \mathrm{OH}=1: 5$ solution at room temperature (RT) for $1 \mathrm{~min}$.
The XPS measurement system (Perkin-Elmer PHI 1600C) consists of a spherical capacitor analyzer and a monochromated Al $K \alpha$ x-ray source ( $h \nu=1486.6 \mathrm{eV})$. The calibration of binding energy of the spectra was done carefully through separate measurements on $\mathrm{Cu} 2 p_{3 / 2}, \operatorname{Ag~} 3 d_{5 / 2}$, and $\mathrm{Au} 4 f_{7 / 2}$ peak positions using standard metal samples. To explore electronic properties of free InGaP surfaces, the unique UHV contactless $C-V$ method was employed. Figure 1 schematically shows its basic setup. An ultranarrow "UHV gap" (200-500 nm) between the upper electrode and the sample surface was set and kept precisely, as shown in Fig. 1(a), by the piezoelectric actuators using capacitance feedback from three parallelism electrodes [Fig. 1(b)]. This UHV gap acts as an ideal insulating layer, and probes electrical properties of free semiconductor surfaces through the conventional $C-V$ assessment. The UHV-gap length was determined by an optical technique. When the UHV-gap length approaches the submicron range, the reflectivity of the laser light becomes less than unity even under the absolute reflection condition, due to the penetration of an evanescent wave. From this reflectivity change, the UHV-gap length can easily be calculated. The area of the measurement electrode is 7.5 $\times 10^{-3} \mathrm{~cm}^{2}$ and the measurement frequency is $500 \mathrm{kHz}$. The stability and sensitivity of the system were checked using the $\mathrm{SiO}_{2} / \mathrm{Si}$ structure prepared by dry oxidation at $1000^{\circ} \mathrm{C}$ as a standard.

A typical Raman spectrum of the MOVPE InGaP layer taken by the backscattering geometry at RT is shown in Fig. 2. Clear peaks corresponding to the InP-like and GaP-like

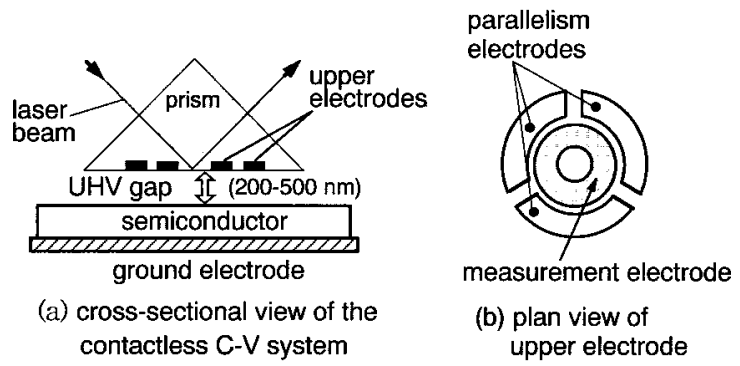

FIG. 1. Schematic illustration of basic setup of the UHV contactless $C-V$ system.

${ }^{\text {a)} E l e c t r o n i c ~ m a i l: ~ h a s h i @ r c i q e . h o k u d a i . a c . j p ~}$ 


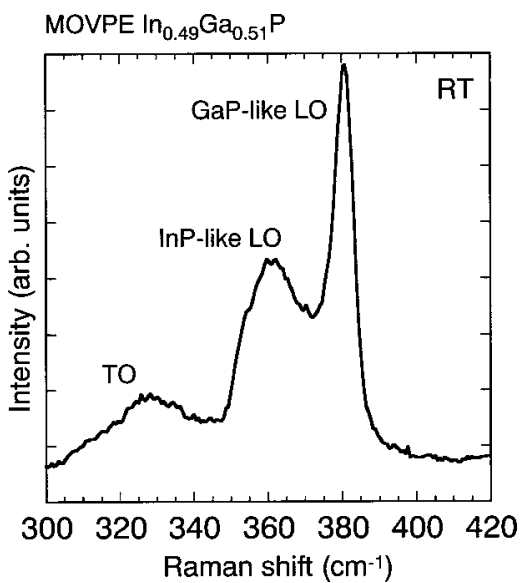

FIG. 2. A typical Raman spectrum of the MOVPE InGaP layer taken by the backscattering geometry at RT.

longitudinal optical phonon frequencies appeared at around $361 \mathrm{~cm}^{-1}$ and $381 \mathrm{~cm}^{-1}$, respectively, similarly to those in literature. ${ }^{8,9}$ Furthermore, deep level transient spectroscopy study showed no pronounced electron trap with the density higher than $1 \times 10^{14} \mathrm{~cm}^{-3}$ in the as-grown layer. The photoluminescence (PL) spectrum showed a sharp band-edge emission from the InGaP layer at $1.85 \mathrm{eV}$ at RT. These results indicated that the present InGaP material has a high bulk crystalline quality. From the PL peak energy, an ordering parameter, $\eta$, was estimated to be 0.3 , using a value of $2.01 \mathrm{eV}$ for the band gap of a completely disordered $\mathrm{InGaP}$ lattice and a value of $0.471 \mathrm{eV}$ for the maximum decrease of a band gap in a completely ordered lattice. ${ }^{6,10}$

The XPS valence-band spectra of the air-exposed and $\mathrm{HCl}$-treated InGaP surfaces are shown in Fig. 3. Photoelectrons were detected using an electron escape angle of $15^{\circ}$, implying that the obtained spectra reflected information from the topmost surface region (within 1.5-2.0 nm). For comparison, the spectrum taken from the $\mathrm{GaP}$ surface after the thermal cleaning in an UHV is also plotted in Fig. 3. The HCl-treated InGaP surfaces showed very similar spectrum to

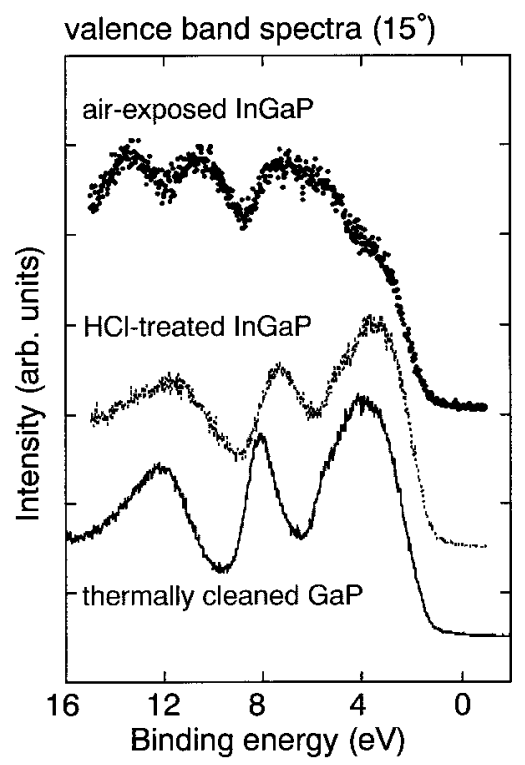

FIG. 3. The XPS valence-band spectra of the air-exposed and HCl-treated InGaP surfaces. Photoelectrons were detected using an electron escape angle

of $15^{\circ}$.
Downloaded 24 Feb 2006 to 133.87.26.100. Redistribution subject to AIP license or copyright, see http://apl.aip.org/apl/copyright.jsp

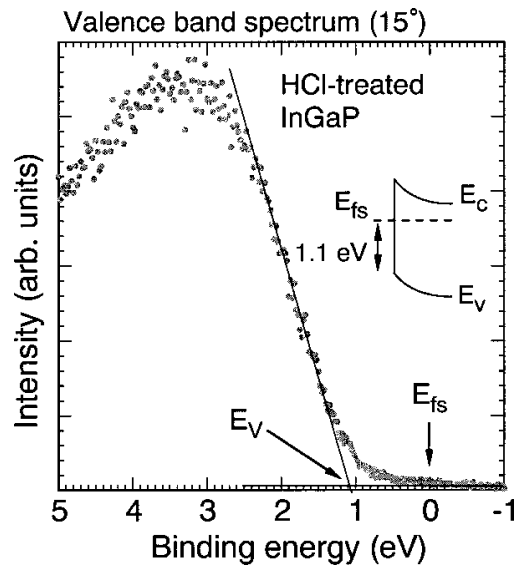

FIG. 4. The photoemission spectrum of the HCl-treated surface near valence-band edge region.

that of the UHV thermally cleaned GaP surface, indicating that it reflected lattice bonds of the host-binary materials. As shown in the upper trace in Fig. 3, on the other hand, the spectrum of the air-exposed surface is very different from that of the chemically treated surface. Detailed XPS analysis revealed $^{11}$ that the air-exposed surfaces included a large amount of natural oxide where the $\mathrm{InPO}_{4}$-like chemical phase was dominant. Thus, the results in Fig. 3 clearly indicate that the electronic properties of the air-exposed InGaP surface was governed by the natural oxide layer. It was also shown ${ }^{11}$ that the $\mathrm{HCl}$ and $\mathrm{HF}$ treatments were effective in reducing natural oxide and in recovering the surface stoichiometry.

To obtain a better insight into electronic properties of InGaP surfaces, the surface Fermi-level position, $E_{\mathrm{fs}}$, was estimated from the analysis on valence-band spectra. Figure 4 shows the photoemission spectrum of the HCl-treated surface near the valence-band edge region. The energy position of the valence-band maximum relative to $E_{\mathrm{fs}}$ was determined by extrapolating a straight line through the leading edge of the spectrum. Thus, the value of $E_{f s}-E_{v}$ was determined to be $1.1 \pm 0.1 \mathrm{eV}$. This indicates a relatively weak band bending of $0.6-0.7 \mathrm{eV}$ at the $\mathrm{HCl}$-treated InGaP surfaces, as shown in the inset of Fig. 4. For the HF-treated surfaces, $E_{f s}-E_{v}=1.0 \pm 0.1 \mathrm{eV}$ was obtained.

Figure 5(a) shows the measured UHV contactless $C-V$ curve and the corresponding surface Fermi-level position, $E_{c}-E_{\mathrm{fs}}$, at given gate bias for the $\mathrm{InGaP}$ surface after the $\mathrm{HCl}$ treatment. The solid line indicates the calculated $\mathrm{C}-\mathrm{V}$ curve based on the accumulation, depletion, and inversion behavior for the metal-insulator-semiconductor structure including the UHV gap as an insulator. ${ }^{12}$ The UHV gap was determined to be $255 \mathrm{~nm}$ whose equivalent thickness to a $\mathrm{SiO}_{2}$ film corresponds to $994 \mathrm{~nm}$. Relatively large gate voltage swing is thus necessary for the change in capacitance from the accumulation to depletion conditions. The measured $C-V$ curve showed more gentle slope in the depletion region as compared with the calculated one. However, the maximum and minimum capacitance values are close to those corresponding to the insulator capacitance and the inversion capacitance, respectively. This indicated that the potential at the $\mathrm{HCl}$-treated InGaP surface can be controlled through almost the entire band gap by the applied gate field, as maniAIP license or copyright, see http://apl.aip.org/apl/copyright.jsp 

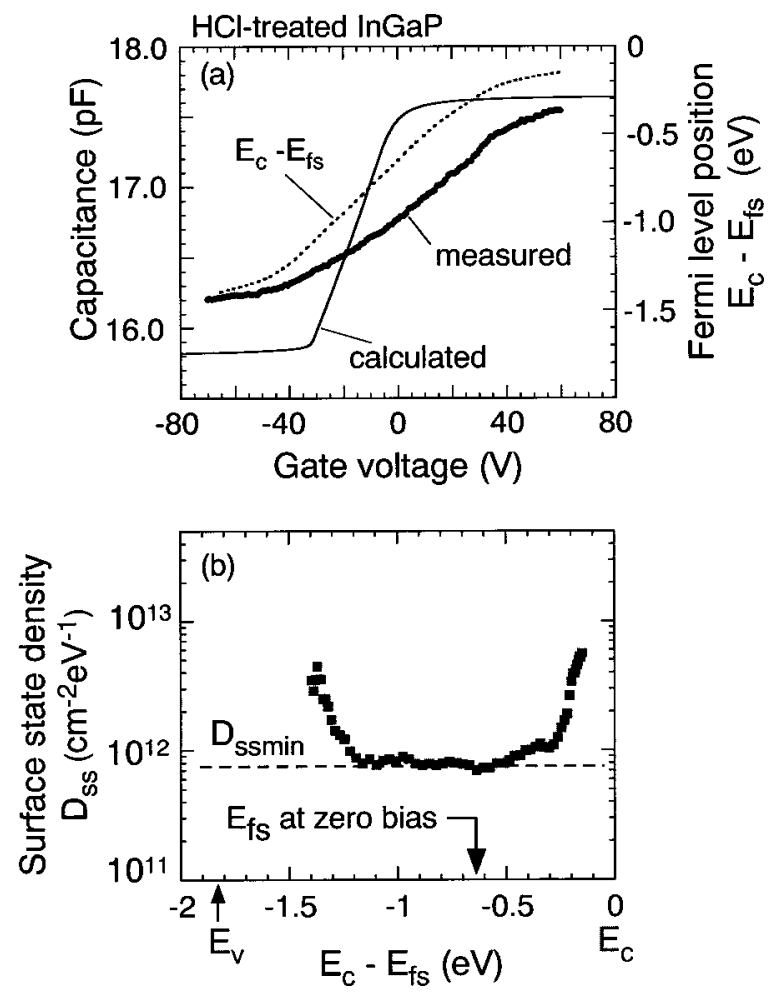

FIG. 5. (a) The measured UHV contactless $C-V$ curve and the corresponding surface Fermi-level position, $E_{c}-E_{\mathrm{fs}}$, for the $\mathrm{InGaP}$ surface after the $\mathrm{HCl}$ treatment. (b) The surface state density $\left(D_{\mathrm{ss}}\right)$ distribution calculated by the Terman method.

fested in the obtained energy positions of surface Fermi level indicated by the dotted line in Fig. 5(a).

The surface state density $\left(D_{\mathrm{ss}}\right)$ distribution calculated by the Terman method ${ }^{12}$ reflected this feature, as shown in Fig. 5(b). The HCl-treated InGaP surface showed a wide $D_{\text {ss }}$ distribution in energy with relatively low $D_{\text {ss }}$ densities, leading to no pronounced Fermi-level pinning effect on the surface. The minimum $D_{\text {ss }}$ value was $8 \times 10^{11} \mathrm{~cm}^{-2} \mathrm{eV}^{-1}$. This probably resulted in the significant passivation effects of an InGaP layer in power GaAs FETs ${ }^{1}$ and $\mathrm{InGaP} / \mathrm{GaAs}$ HBTs. ${ }^{2}$

It is shown in the $D_{\text {ss }}$ distribution that gap states near the valence-band edge have higher densities than those near the conduction-band edge. The tail detected in the XPS valenceband spectrum (Fig. 4) seems to reflect such a feature in the $D_{\mathrm{ss}}$ distribution. The $E_{\mathrm{fs}}$ position at zero bias was found at $E_{V}+1.2 \mathrm{eV}\left(E_{c}-0.65 \mathrm{eV}\right)$, reasonably in agreement with the value of $E_{V}+1.1 \mathrm{eV}$ obtained from the XPS analysis mentioned herein. Tersoff, ${ }^{13}$ Cordona and Christensen, ${ }^{14}$ and Mönch $^{15}$ have calculated the charge neutrality energies, which are expected to control the lineup at various kinds of semiconductor interfaces, for major elementary semiconductors and binary compound semiconductors. They predicted the values of $E_{V}+0.81 \mathrm{eV}$ (Refs. 12 and 13) and $E_{V}$ $+0.83 \mathrm{eV}$ (Ref. 14) for GaP, and $E_{V}+0.76 \mathrm{eV}$ (Refs. 12 and 13) and $E_{V}+0.86 \mathrm{eV}$ (Ref. 14) for InP. The present results indicate that the charge neutrality level relative to the valence-band maximum for InGaP lies at higher energies than those of its host-binary compounds. This may be related to the sublattice ordering effects.

In summary, the effectiveness and availability of the UHV contactless $C-V$ method for directly probing the electronic properties of the free InGaP surfaces were demonstrated. The HCl-treated surface showed a wide U-shaped surface state density distribution in energy with relatively low densities, leading to no pronounced Fermi-level pinning effect on the surface. The minimum $D_{\text {ss }}$ value was determined to be $8 \times 10^{11} \mathrm{~cm}^{-2} \mathrm{eV}^{-1}$. The surface Fermi-level position was found at $1.2 \mathrm{eV}$ above the valence-band maximum, consistent with the results obtained from the XPS valence-band spectra. The basic information on the surface electronic properties obtained here is very useful for the design of suitable surface passivation structures for further advanced HBTs and high-electron mobility transistors.

The author would like to thank Dr. T. Saitoh, Hitachi Cable Ltd., for supplying the MOVPE InGaP wafers. This work was partly supported by a grant from the Support Center for Advanced Telecommunications Technology Research (SCAT).

${ }^{1}$ T. Tanaka, H. Furukawa, K. Miyatsuji, and D. Ueda, Mater. Res. Soc. Symp. Proc. 573, 239 (1999).

${ }^{2}$ Y. F. Yang, C. C. Hsu, and E. S. Yang, IEEE Trans. Electron Devices 41, 643 (1994).

${ }^{3}$ N. Pan, J. Elliot, M. Knowles, D. P. Vu, K. Kishimoto, J. K. Twynam, H. Sato, M. T. Fresina, and G. E. Stillman, IEEE Electron Device Lett. 19, 115 (1998).

${ }^{4}$ B. P. Yan, Y. F. Yang, C. C. Hsu, H. B. Lo, and E. S. Yang, Microelectron. Reliab. 41, 1959 (2001)

${ }^{5}$ A. Gomyo, T. Suzuki, and S. Iijima, Phys. Rev. Lett. 60, 2645 (1988).

${ }^{6}$ K. A. Mäder and A. Zunger, Phys. Rev. B 51, 10462 (1995).

${ }^{7}$ S. Froyen, A. Zunger, and A. Mascarenhas, Appl. Phys. Lett. 68, 2852 (1996).

${ }^{8}$ F. Alsina, N. Mestres, J. Pascual, C. Geng, P. Ernst, and F. Scholz, Phys. Rev. B 53, 12994 (1996).

${ }^{9}$ H. M. Cheong, A. Mascarenhas, P. Ernst, and C. Geng, Phys. Rev. B 56, 1882 (1997)

${ }^{10}$ P. Ernst, C. Geng, F. Scholz, H. Schweizer, Y. Zhang, and A. Mascarenhas, Appl. Phys. Lett. 67, 2347 (1995).

${ }^{11}$ T. Hashizume and T. Saitoh, Appl. Phys. Lett. 78, 2318 (2001).

${ }^{12}$ E. H. Nicollian and J. R. Brews, MOS Physics and Technology (Wiley, New York, 1982), Chap. 8.

${ }^{13}$ J. Tersoff, J. Vac. Sci. Technol. B 4, 1066 (1986).

${ }^{14}$ M. Cordona and N. E. Christensen, Phys. Rev. B 35, 6182 (1987).

${ }^{15}$ W. Mönch, Appl. Surf. Sci. 117, 380 (1997). 